\title{
An ultrathin transparent metamaterial polarization transformer based on a twist-split-ring resonator
}

\author{
Yongzhi Cheng $\cdot$ Yan Nie $\cdot$ Xian Wang $\cdot$ Rongzhou Gong
}

Received: 5 October 2012 / Accepted: 3 January 2013 / Published online: 19 January 2013

(C) The Author(s) 2013. This article is published with open access at Springerlink.com

\begin{abstract}
In this paper, an ultrathin transparent metamaterial polarization transformer using a circular twist-split-ring resonator (TSRR) was proposed and investigated experimentally and numerically. The experimental and simulated results exhibit an asymmetric transmission only for forward and backward propagating linearly polarized waves. An incident linearly polarized wave can convert its polarization nearly completely to the cross direction after transmission under certain conditions. The simulated spatial evolution of the electric field further indicates that the twist structure functions as a perfect polarization transformer at certain frequencies.
\end{abstract}

\section{Introduction}

Electromagnetic (EM) metamaterials (MMs) have attracted enormous interest in recent decades due to their unique responses to EM radiation, which are generally not encountered in their natural forms [1, 2]. MMs are usually periodically arranged with artificial materials that can be composed of dielectric elements or structured metallic components $[2,3]$. Polarization is an important characteristic of EM waves and could be applicable in many areas, such as antennas, astronavigation, and communication [4-8]. Thus, it is highly desirable to efficiently control the polarization of EM waves [9]. Conventionally, optical gratings and dichroic crystals can be used to convert polarization by employing, e.g., the Brewster and birefringence effects [10]. How-

Y. Cheng $\cdot$ Y. Nie $\cdot$ X. Wang $\cdot$ R. Gong $(\bowtie)$

School of Optical and Electronic Information,

Huazhong University of Science and Technology, Wuhan 430074,

China

e-mail: rzhgong@mail.hust.edu.cn ever, the conversion efficiency is very low. Recently, chiral metamaterials (CMMs) have attracted much attention, due to their optical activity, circular or elliptical dichroism, and negative index [11-20]. CMMs are artificial materials that lack any planes of mirror symmetry; they exhibit crosscoupling between the electric and magnetic fields at resonance $[10,15]$. Thus, the similar design of chiral MMs could be used to convert polarization of incident EM waves, resulting in, e.g., giant optical activity [15] and polarization rotation [17]. The asymmetric transmission (AT) of polarized light is an important research aspect of planar CMMs and has become a hot topic in recent years [18-25]. The AT effect of circular or linear polarization has been studied for different MM structures, where the partial conversion of the incident EM wave is asymmetric for opposite propagation directions, which mainly originates from the interaction of EM radiation with the structural two-dimensional (2D) chirality in the MMs [23-27]. Thus, we can manipulate the polarization of EM waves by employing the AT effect, such as converting its polarization completely to the cross direction, and realizing circular or elliptical polarization after transmission under certain conditions [15, 23-27]. Menzel et al. and Kang et al. introduced the design criterion of AT for polarized EM radiation theoretically with a $4 \times 4$ matrix analysis based on the classical model of optical activity [21, 23, 24].

In this paper, we demonstrate experimentally and numerically an AT phenomenon for linearly polarized EM waves only and a linear polarization transformation. Based on the design criterion of the AT effect of MMs, the designed MM polarization transformer is composed of $90^{\circ}$ twist-split-ring resonators (TSRRs) on both sides of a dielectric slab. A polarization conversion ratio (PCR) of $95 \%$ in the experiment and $97 \%$ in simulation can be achieved. Calculations on the spatial evolution of field distributions provide an intuitive 
picture of the interlayer coupling for polarization transformation inside the slab. Furthermore, when using a loss-free dielectric substrate as the middle spacer, the AT parameter can achieve a value of nearly unity.

\section{Design, simulation, and experiment}

First, for the Cartesian coordinates $x-y-z$, considering an incident EM field $\boldsymbol{E}^{i}$ propagating in the $+z$ direction, we can define expressions for the electric field vector of the incident and transmitted wave along the $x$-polarized and $y$-polarized directions as follows:

$\boldsymbol{E}^{i}(r, t)=\left(\begin{array}{c}E_{x}^{i} \\ E_{y}^{i}\end{array}\right) e^{i(k z-\omega t)}$

$\boldsymbol{E}^{t}(r, t)=\left(\begin{array}{c}E_{x}^{t} \\ E_{y}^{t}\end{array}\right) e^{i(k z-\omega t)}$

where $\omega, k, E_{x}$, and $E_{y}$ represent the angular frequency, wave vector, and complex amplitudes of the EM wave, respectively. To better understand the cross-polarization conversion of the designed MM structure, the transmission/reflection coefficient $t_{i j}^{f(b)}=E_{i}^{t} / E_{j}^{i} / r_{i j}^{f(b)}=E_{i}^{r} / E_{j}^{i}$ is defined in terms of the complex amplitude of the electric field, with the first and the second subscripts $i$ and $j$ denoting the polarized states of the transmitted/reflected wave and incident wave components, respectively. The superscripts $f$ and $b$ denote forward propagation (along the $+z$ direction) and backward propagation (along the $-z$ direction) of the EM wave, and a special linear base with base vectors parallel to the coordinate axes, i.e., decomposing the field into $x$ - and $y$-polarized waves, respectively. Thus, we can use the Jones matrix (transmission matrix, also called $T$ matrix) formulations and the reciprocity theorem to obtain the transmission propagation for a linear polarized wave in the $+z$ and $-z$ directions [14]:

$\left(\begin{array}{c}t_{x}^{f} \\ t_{y}^{f}\end{array}\right)=T_{l i}^{f}\left(\begin{array}{c}E_{x}^{i} \\ E_{y}^{i}\end{array}\right)=\left(\begin{array}{ll}t_{x x} & t_{x y} \\ t_{y x} & t_{y y}\end{array}\right)\left(\begin{array}{c}E_{x}^{i} \\ E_{y}^{i}\end{array}\right)$

$\left(\begin{array}{c}t_{x}^{b} \\ t_{y}^{b}\end{array}\right)=t_{l i}^{b}\left(\begin{array}{c}E_{x}^{i} \\ E_{y}^{i}\end{array}\right)=\left(\begin{array}{cc}t_{x x} & -t_{y x} \\ -t_{x y} & t_{y y}\end{array}\right)\left(\begin{array}{c}E_{x}^{i} \\ E_{y}^{i}\end{array}\right)$

The subscript $l i$ indicates the linear polarized wave. Thus, the $T$ matrices for propagation in the $+z$ and $-z$ directions for a linear polarization basis, $t_{l i}^{f}$ and $t_{l i}^{b}$, can be expressed as:

$t_{l i}^{f}=\left(\begin{array}{cc}t_{x x} & t_{x y} \\ t_{y x} & t_{y y}\end{array}\right)$ $t_{l i}^{b}=\left(\begin{array}{cc}t_{x x} & -t_{y x} \\ -t_{x y} & t_{y y}\end{array}\right)$

where $t_{x x}$ and $t_{y y}$ indicate the co-polarization transmission coefficient, and $t_{x y}$ and $t_{y x}$ indicate the cross-polarization transmission coefficient. We use the parameter $\gamma$ to represent the PCR of the transmitted wave in different polarization directions ( $x$-polarization and $y$-polarization) for the incident waves; this parameter can be defined as follows:

$\gamma_{x}=\left|t_{y x}\right|^{2} /\left(\left|t_{y x}\right|^{2}+\left|t_{x x}\right|^{2}+\left|r_{y x}\right|^{2}+\left|r_{x x}\right|^{2}\right)$

$\gamma_{y}=\left|t_{x y}\right|^{2} /\left(\left|t_{x y}\right|^{2}+\left|t_{y y}\right|^{2}+\left|r_{x y}\right|^{2}+\left|r_{y y}\right|^{2}\right)$

If, without absorption and diffractions, the denominator of the above two equations is 1 , then we can get the PCR parameters $\gamma^{x}=\left|t_{y x}\right|^{2}$ and $\gamma^{y}=\left|t_{x y}\right|^{2}$, respectively. The conversion relation of the transmission coefficients of linearly and circularly polarized waves for propagation in the $+z$ direction can be given as:

$$
\begin{aligned}
t & =\left(\begin{array}{ll}
t_{++} & t_{+-} \\
t_{-+} & t_{--}
\end{array}\right) \\
& =\frac{1}{2}\left(\begin{array}{ll}
\left(t_{x x}+t_{y y}\right)+i\left(t_{x y}-t_{y x}\right) & \left(t_{x x}-t_{y y}\right)-i\left(t_{x y}+t_{y x}\right) \\
\left(t_{x x}-t_{y y}\right)+i\left(t_{x y}+t_{y x}\right) & \left(t_{x x}+t_{y y}\right)-i\left(t_{x y}-t_{y x}\right)
\end{array}\right)
\end{aligned}
$$

Generally, the AT effect for both the linearly and circularly polarized waves is characterized by the parameter $\Delta$, which is defined as the difference between the transmittances in the two opposite propagation directions $(+z$ and $-z$ ) and is used to represent the degree of the AT [23-25]. Thus, the AT parameter $\Delta$ for the linearly and circularly polarized waves can be expressed as:

$\Delta_{l i}^{x}=\left|t_{y x}\right|^{2}-\left|t_{x y}\right|^{2}=-\Delta_{l i}^{y}$

$\Delta_{l i}^{-}=\left|t_{+-}\right|^{2}-\left|t_{-+}\right|^{2}=-\Delta_{l i}^{+}$

According to the above classical formulations of optical activity, we can find the AT of transmitted waves for linear polarization only can be achieved [21]:

$\Delta_{l i}^{x}=-\Delta_{l i}^{y} \neq 0$

$\Delta_{l i}^{-}=-\Delta_{l i}^{+}=0$

Obviously, the AT effect depends on the specific polarization base. For both bases given here it is exclusively determined by the difference between the off-diagonal elements of the respective $T$ matrices. Additionally, note that for an arbitrary base the diagonal elements of the $T$ matrix for different propagation directions are not identical and will also 


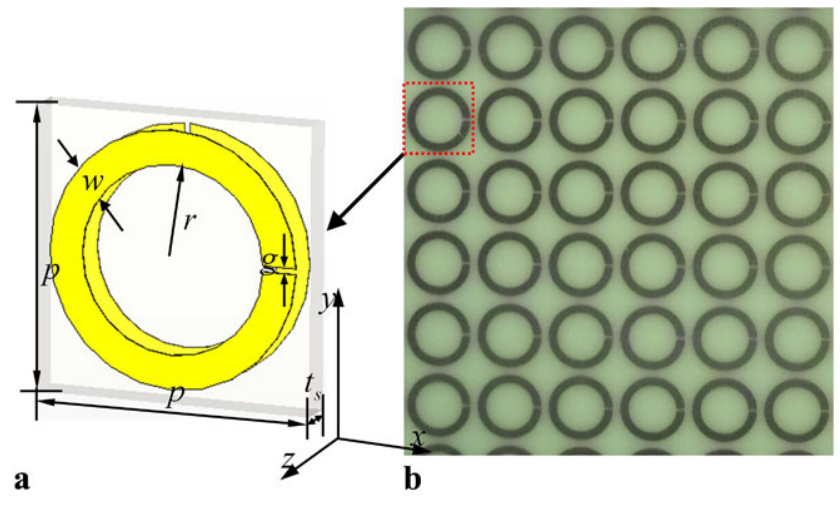

Fig. 1 (a) Unit cell of bilayer TSRR structure. (b) Photograph of the fabricated sample

contribute to AT [22]. Thus, the transmission elements of co-polarization and cross-polarization should satisfy the following conditions:

$\left|t_{y x}\right| \neq\left|t_{x y}\right|$

$\left|t_{x x}\right|=\left|t_{y y}\right|$

Based on the above analysis, to achieve polarization transformation, we can design a similar CMM structure. The metallic resonant structure in each layer has the same arbitrary pattern; however, when the first layer structure is fixed, the structure in the second layer should be rotated clockwise $90^{\circ}$ along the propagation direction of the EM wave. Thus, any planes of mirror symmetry can be broken.

Here, we employ a simple circular split-ring resonator (SRR) as the resonant structure. The unit cell of the designed MM consists of two metallic SRRs on both sides of a dielectric substrate twisted $90^{\circ}$ to each other, which is different than the design of a negative refractive index MM [28]. Figure 1(a) shows the schematic diagram of the unit cell; the metallic pattern in the front and back layers is symmetric along the $x$ axis and $y$ axis, respectively. This design will satisfy the requirements of (8a), while also facilitating the achievement of an efficient coupling between the two chiral planar MMs and an enhanced EM response [29]. Finally, the mirror symmetry of the whole structure is broken in the propagation $z$ direction and in the $x-y$ plane, due to the two copper patterns separated by the middle dielectric spacer. The geometry dimension parameters are, in millimeters: $p=10, r=3.3, g=0.2, w=1.2, t_{s}=1.2$. The metallic layers on both sides were modeled as a $0.036 \mathrm{~mm}$ copper film with an electric conductivity $\sigma=5.8 \times 10^{7} \mathrm{~S} / \mathrm{m}$. An FR-4 (lossy) substrate with a permittivity of 3.6 and a loss tangent of 0.025 was selected as the dielectric spacer. The simulation has been performed based on the standard finite difference time domain (FDTD) method by using CST Microwave Studio's Frequency Domain Solver. The periodic boundary conditions are applied to the $x$ and $y$ directions and the absorbing boundary conditions to the $z$ direction as well.

For the experiments, the designed structures were fabricated into a $20 \times 20$ unit cell sample $(200 \mathrm{~mm} \times 200 \mathrm{~mm}$ $\times 1.272 \mathrm{~mm}$ ) using the conventional printed circuit board (PCB) process with $36 \mu \mathrm{m}$ thick copper patterns on both sides of a FR-4 (lossy) substrate. A photograph of a portion of a fabricated circular TSRR structure MM sample is shown in Fig. 1(b). The complex transmission/reflection coefficients of the experimental measurements were carried out in an EM anechoic chamber [27]. An Agilent PNA-X N5244A vector network analyzer connected to two standard gain broadband linearly polarized horn antennas that produced microwaves in the range of $7-12 \mathrm{GHz}$ was used to measure the MM sample. All components of the EM wave transmission/reflection (the complex Jones matrix) for different polarizations were measured by changing the orientation of the two horn antennas.

\section{Results and discussion}

Based on the preceding simulations and experiments, we can get the transmission/reflection coefficients (the complex Jones matrix) as shown in Fig. 2. This figure shows the simulated and measured spectra for the four transmission/reflection matrix elements of the designed slab for propagation in the backward $(-z)$ direction. The experimental results are in excellent agreement with the simulations across the whole frequency range. From Figs. 2(a) and (b), when the propagation is along the $-z$ direction of the incident linear polarization wave, the co-polarization transmission $t_{x x}$ and $t_{y y}$ always remain the same (e.g., $t_{x x}=t_{y y}$ ), while the cross-polarization components $t_{x y}$ and $t_{y x}$ differ significantly across the whole frequency range both in the experiment and the simulation. These results are in agreement with our analysis in (8a) and (8b), clearly leading to a strong AT effect for linear polarization. The amplitudes for the co-polarization transmission reduce to a minimum of about 0.1 at $9.9 \mathrm{GHz}$. One can clearly observe that the amplitude of the cross-polarization transmission $t_{x y}$ achieves a maximum of 0.74 in the experiment and 0.77 in the simulation around the resonance frequency of $9.65 \mathrm{GHz}$, while $t_{y x}$ is very small and stays below 0.1 in the entire frequency range.

The remarkable difference between the two cross-polarization transmissions may contribute to the polarization conversion of the designed structure. Thus, when the $y$-polarized ( $x$-polarized) wave is normally incident to the structure along the $-z(+z)$ direction, the wave is well coupled to the structure and is converted mostly to the $x$-polarized ( $y$-polarized) wave due to the cross-coupling between the two metallic layers when passing through the designed MM. 
Fig. 2 (a), (c) Measured and (b), (d) calculated transmission and reflection spectra of the four matrix components for backward propagation
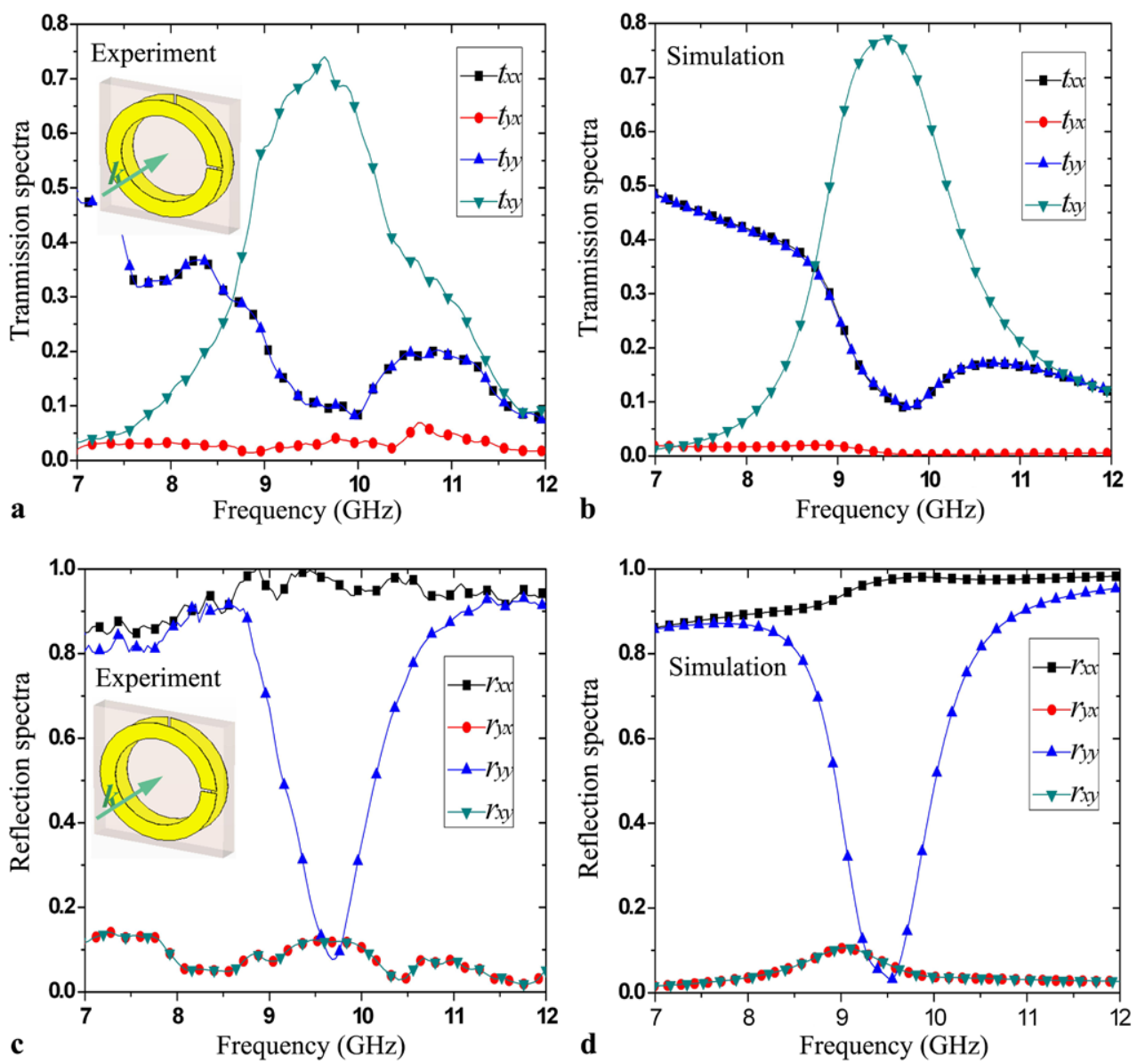

However, along the opposite direction, the $y$-polarized $(x$ polarized) wave can hardly be coupled to the structure, resulting in a weak transmission $[18,25]$. In other words, the incident $y$-polarized ( $x$-polarized) wave is well converted to a transmitted $x$-polarized ( $y$-polarized) wave when passing through the designed MM, while the incident $x$-polarized ( $y$-polarized) wave will be mostly forbidden to transmit for propagation along the $-z(+z)$ direction. Thus, the designed MM can be used as a transparent polarization transformer for linearly polarized EM radiation. Figures 2(c) and (d) show the experimental and simulated reflection (the complex Jones matrix) for propagation along the $-z$ direction; the cross-polarization reflections $r_{x y}$ and $r_{y x}$ are very small and below 0.15 in the entire frequency range. While the copolarization reflection $r_{y y}$ reduces to a minimum of about 0.1 at a frequency of $9.5 \mathrm{GHz}, r_{x x}$ is near unity both in the experiment and the simulation across the whole frequency range. These results of the reflection further verify that the incident $x$-polarized ( $y$-polarized) wave will be forbidden to transmit for propagation along the $-z(+z)$ direction. That is, the coupling of the split rings among each other is very low for this polarization, and nearly without magnetic coupling, resulting in a high reflectivity, and no polarization transformation can be observed.
According to (6a) and (6b), we calculated the AT parameter $\Delta$ for both linear and circular polarization bases; the results are illustrated in Fig. 3. One can observe that the amplitude of the parameter $\Delta$ for linear polarization achieves a maximum of 0.55 in the experiments and 0.59 in the simulations around $9.65 \mathrm{GHz}$. The parameter $\Delta$ for circular polarizations is near zero across the whole frequency range, which indicates that there is no AT for either the left or the right circularly polarized waves due to the specific asymmetry in the designed structure. These results are in agreement with our theoretical prediction in (7a) and (7b), and also further verify the AT effect of our proposed structure.

Generally, the parameter $\gamma$ can be used to measure the polarization conversion ratio of the designed MM for linearly polarized EM radiation. The PCR parameter $\gamma$ of the proposed structure, calculated using (4a) and (4b) for the linear polarization base for backward propagation, is illustrated in Fig. 4. From this figure, one can see clearly that the parameter $\gamma$ for the incident $y$-polarized wave was achieved to a maximum of $95 \%$ in the experiment and $97 \%$ in the simulation around the resonant frequency of $9.65 \mathrm{GHz}$ for backward propagation. However, the parameter $\gamma$ for the incident $x$-polarized wave is near zero for both the experiment and the simulation across the whole frequency range 
Fig. 3 (a) Measured and (b) calculated AT parameter $\Delta$ for linear and circular polarization bases
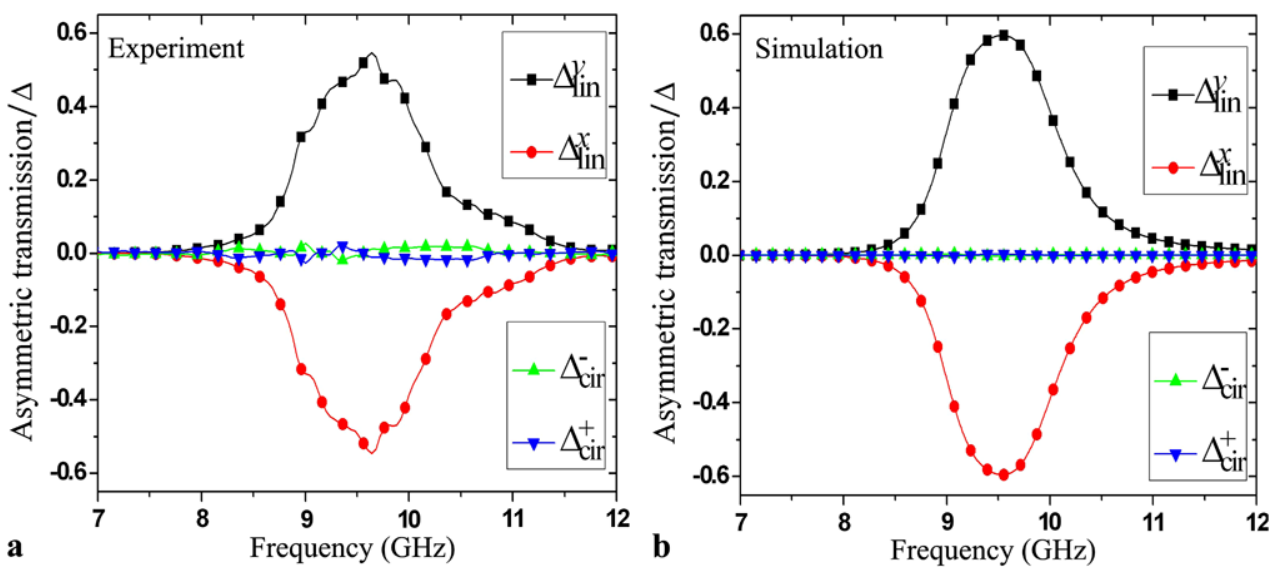

Fig. 4 (a) Measured and (b) calculated PCR parameter $\gamma$ for different linear polarization bases for backward propagation
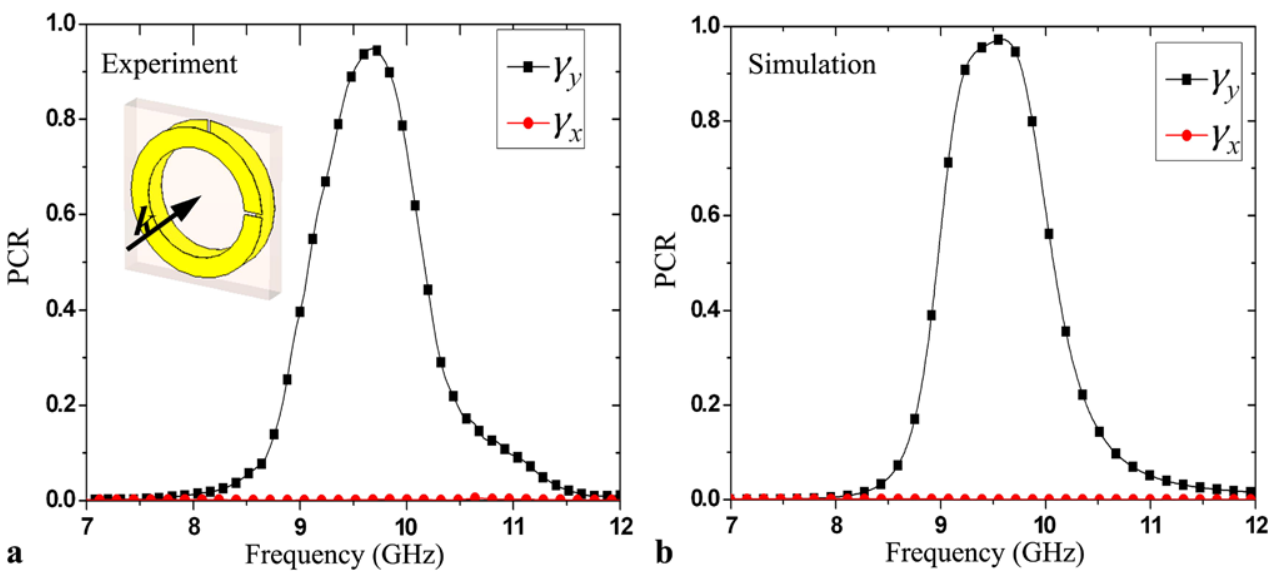

for backward propagation. These results indicate that an incident linearly polarized wave passing through the designed MM can nearly perfectly convert its polarization to the cross direction after transmission under certain conditions.

To better understand the physical insight of the polarization transformation that is associated with the designed MM, we have calculated snapshots of the EM wave evolution when propagating through the structure around the resonant frequency. Figure 5 illustrates six snapshots of the electric field distribution for a $y$-polarized and $x$-polarized wave passing through the designed slab backward. These snapshots correspond to the electric field distributions for the EM wave in the incoming and outgoing regime as well as inside the designed MM. It can be seen clearly that the outgoing waves are always $x$-polarized (see Figs. 5(c) and (f)), even when the incident wave is $y$-polarized (see Fig. 5(a)) for backward transmission at $9.6 \mathrm{GHz}$. We can obtain a reasonable explanation from the theoretical study of SRR structure in Ref. [30], which demonstrated the interesting bianisotropic responses to EM waves. When the $y$-polarized wave is incident along the backward direction, the fundamental electric resonance mode (similar to the electric dipole resonance mode) of the first circular SRR can be excited efficiently at the resonance with a strong EM wave being coupled into the designed MM. Then the near-field coupling between the first and the second SRRs (similar to the magnetic dipole mode) results in an obvious transmitted wave, but the polarization has been converted to an $x$-polarized form. However, when the incident $x$-polarized wave passes through the designed MM for backward propagation, the field strength inside the structure remains high (see Fig. 5(e)), and the incident wave is blocked at the entrance. Thus, the EM wave coupled into the MM is very weak, and results in a very small transmission without polarization transformation (see Figs. 5(d) and (f)). These features of near-field coupling between the metallic layers of our proposed MM structure could further validate its function as a perfect ultrathin (the thickness of the MM is smaller than $\lambda / 26$, where $\lambda$ is the operating wavelength) transparent polarization transformer.

Going a step further, from the above analysis of experimental and simulated results of the MM polarization transformer for linearly polarized EM radiation, we find that the maximal value of the cross-polarization transmission $t_{x y}$ is relatively small (the experimental and simulated values are 0.74 and 0.77 , respectively) at resonance for backward propagation, which is mainly due to the dielectric loss of the FR-4 substrate. If we use a loss-free dielectric sub- 
Fig. 5 Snapshots of the electric field strength at resonance for $y$-polarized $((\mathbf{a})-(\mathbf{c}))$ and $x$-polarized ((d)-(f)) incident plane waves along the $-z$ direction. (a) and (d): at $z=10 \mathrm{~mm}$ of the incoming regime; (b) and (e): at $z=0 \mathrm{~mm}$ within the middle dielectric layer; (c) and (f): at $z=-10 \mathrm{~mm}$ of the outgoing regime. The electric field strength, normalized to that of the incident wave, is illustrated by the color of the arrows
Fig. 6 (a) The calculated transmission spectra of the four matrix components for backward propagation. (b) Calculated AT parameter $\Delta$ for linear and circular polarization bases
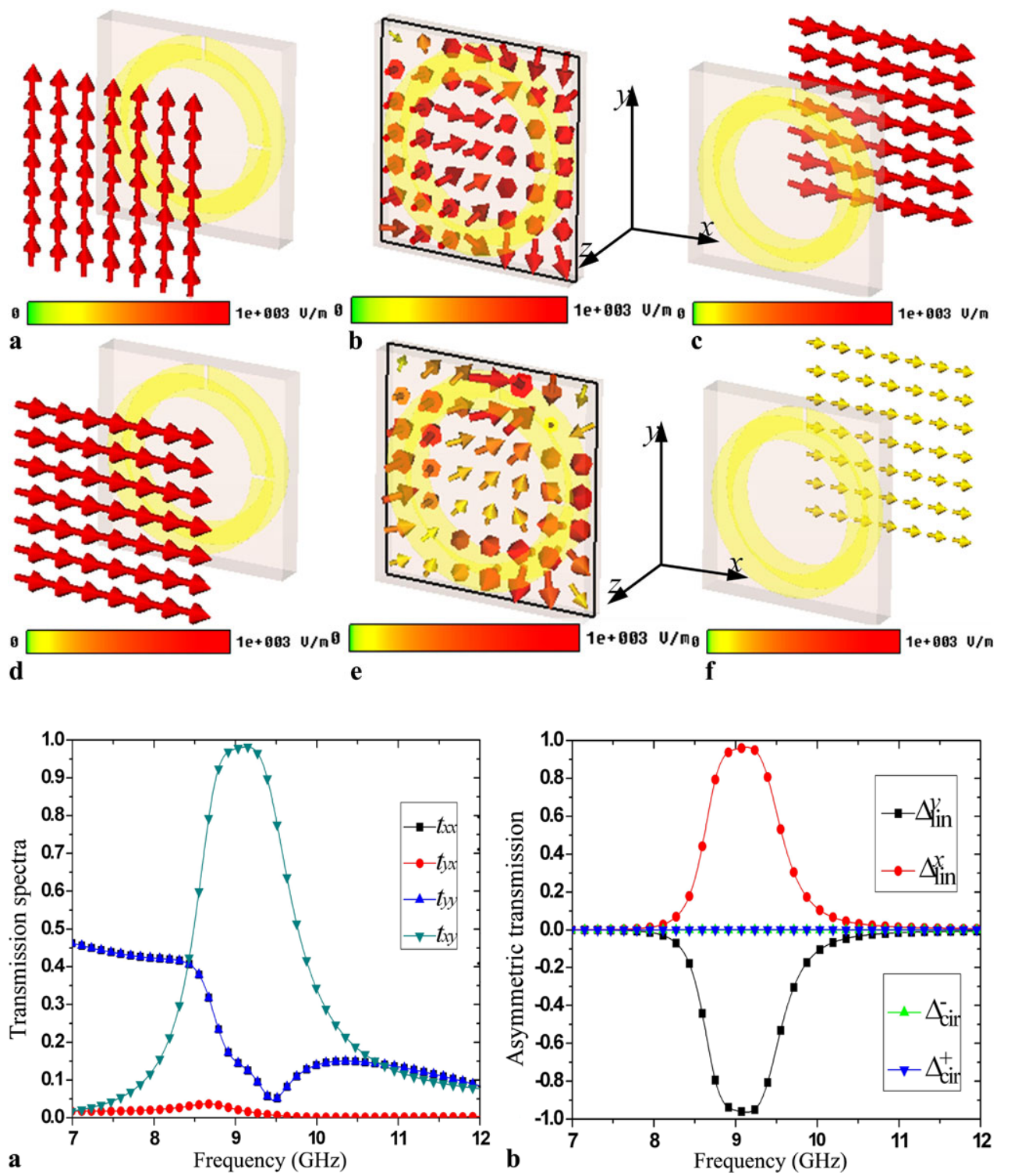

strate as the middle spacer, the maximal value of the crosspolarization transmission $t_{x y}$ could achieve near unity for backward propagation. Figure 6 shows the simulated transmission spectra for back propagation and the AT parameter when using the loss-free FR-4 substrate. One can observe that the maximal value of cross-polarization transmission $t_{x y}$ and the AT parameter achieve values of 0.99 and 0.98 at a resonant frequency of $9.1 \mathrm{GHz}$, respectively, which verifies our previous predictions. Therefore, we can obtain a high performance transparent MM polarization transformer by using a loss-free dielectric substrate.

\section{Conclusion}

In conclusion, we first analyzed the design criterion for the AT effect of MMs, and then demonstrated experimentally and numerically an AT phenomenon for linearly polarized EM waves only and an ultrathin linear polarization transformer based on a TSRR-structured MM with a thickness smaller than $\lambda / 26$. An incident linearly polarized wave can convert its polarization nearly completely to the cross direction after transmission under certain conditions. The PCR was achieved to a maximum of $95 \%$ in the experiment and $97 \%$ in the simulation around a resonance frequency of 9.65 GHz. The calculated snapshots of the EM wave evolution of wave fields propagating inside the structure illustrate the physical mechanism of the AT effect and polarization transformation of our design. Further simulated results indicate that we can obtain a high performance MM polarization transformer by using a loss-free or low-loss dielectric substrate. Such a design may find potential application in optical isolators, microwave wave plates, or other EM control devices. 
Acknowledgements This work is supported by the National Natural Science Foundation of China (51207060).

Open Access This article is distributed under the terms of the Creative Commons Attribution License which permits any use, distribution, and reproduction in any medium, provided the original author(s) and the source are credited.

\section{References}

1. V.G. Veselago, Sov. Phys. Usp. 10, 509 (1968)

2. D.R. Smith, W.J. Padilla, D.C. Vier, S.C. Nemat-Nasser, S. Schultz, Phys. Rev. Lett. 84, 4184 (2000)

3. R.A. Shelby, D.R. Smith, S. Schultz, Science 292, 77 (2001)

4. J.D. Jackson, Classical Electrodynamics, 3rd edn. (Wiley, New York, 1999)

5. M. Born, E. Wolf, Principles of Optics (Cambridge University Press, Cambridge, 1999)

6. R.W. Ziolkowski, A. Erentok, IEEE Trans. Antennas Propag. 54(7), 2113 (2006)

7. S. Tretyakov, I. Nefedov, A. Sihvola, S. Maslovski, C. Simovski, J. Electromagn. Waves Appl. 17(5), 695 (2003)

8. J.B. Pendry, Science 306(5700), 1353 (2004)

9. J.D. Jackson, Classical Electrodynamics, 3rd edn. (Wiley, New York, 1999)

10. A.V. Rogacheva, V.A. Fedotov, A.S. Schwanecke, N.I. Zheludev, Phys. Rev. Lett. 97(17), 177401 (2006)

11. H. Liu, D.A. Genov, D.M. Wu, Y.M. Liu, Z.W. Liu, C. Sun, S.N. Zhu, X. Zhang, Phys. Rev. B 76(7), 073101 (2007)

12. T.Q. Li, H. Liu, T. Li, S.M. Wang, F.M. Wang, R.X. Wu, P. Chen, S.N. Zhu, X. Zhang, Appl. Phys. Lett. 92(13), 131111 (2008)
13. M. Decker, S. Linden, M. Wegener, Opt. Lett. 34(10), 1579 (2009)

14. R.J. Singh, E. Plum, W.L. Zhang, N.I. Zheludev, Opt. Express 18(13), 13425 (2010)

15. B. Wang, T. Koschny, M. Kafesaki, C.M. Soukoulis, J. Opt. A, Pure Appl. Opt. 11, 114003 (2009)

16. J.Y. Chin, M. Lu, T.J. Cui, Appl. Phys. Lett. 93, 251903 (2008)

17. Y. Ye, S. He, Appl. Phys. Lett. 96, 203501 (2010)

18. J. Han, H.Q. Li, Y.C. Fan, Z.Y. Wei, C. Wu, Y. Cao, X. Yu, F. Li, Z.S. Wang, Appl. Phys. Lett. 98, 151908 (2011)

19. R. Singh, E. Plum, C. Menzel, C. Rockstuhl, A.K. Azad, R.A. Cheville, F. Lederer, W. Zhang, N.I. Zheludev, Phys. Rev. B 80(15), 53104 (2009)

20. V.A. Fedotov, P.L. Mladyonov, S.L. Prosvirnin, A.V. Rogacheva, Y. Chen, N.I. Zheludev, Phys. Rev. Lett. 97(16), 167401 (2006)

21. C. Menzel, C. Rockstuhl, F. Lederer, Phys. Rev. A 82, 053811 (2010)

22. A.M. Mutlu, E.A. Andriy, E. Serebryannikov, E. Ozbay, Opt. Express 19(15), 14290 (2011)

23. C. Menzel, C. Helgert, C. Rockstuhl, E.-B. Kley, A. Tunnermann, T. Pertsch, F. Lederer, Phys. Rev. Lett. 104, 253902 (2010)

24. M. Kang, J. Chen, H. Cui, Y. Li, H. Wang, Opt. Express 19, 8347 (2011)

25. C. Huang, Y. Feng, J. Zhao, Z. Wang, T. Jiang, Phys. Rev. B 85(19), 195131 (2012)

26. C. Huang, J. Zhao, T. Jiang, Y. Feng, J. Electromagn. Waves Appl. 26, $1192(2012)$

27. C. Huang, X. Ma, M. Pu, G. Yi, Y. Wang, X. Luo, Opt. Commun. (2012). doi:10.1016/j.optcom.2012.10.046

28. Y.Z. Cheng, H.L. Yang, Y. Nie, R.Z. Gong, Z.Z. Cheng, Appl. Phys. A, Mater. Sci. Process. 103, 989 (2011)

29. Y.Z. Cheng, H.L. Yang, J. Appl. Phys. 108(3), 034906 (2010)

30. L. Zhou, S.T. Chui, Phys. Rev. B 74, 035419 (2006) 